Short Communication

\title{
ON THE FILTERING OF INTERSEGMENTAL LOADS DURING RUNNING
}

\author{
W. Brent Edwards ${ }^{1}$, Karen L Troy ${ }^{1}$, and Timothy R. Derrick ${ }^{2}$ \\ ${ }^{1}$ Department of Kinesiology and Nutrition, University of Illinois at Chicago, \\ Chicago, IL 60612, USA \\ ${ }^{2}$ Department of Kinesiology, Iowa State University, \\ Ames, IA 50011, USA
}

Funding: Unfunded research project

Acknowledgements: The authors thank Ton van den Bogert for making his inverse dynamics benchmark data available and for helpful discussions during manuscript preparation.

Corresponding Author:

W. Brent Edwards

University of Illinois at Chicago

Department of Kinesiology and Nutrition

1919 W. Taylor Street

650 AHSB, M/C 517

Chicago, IL 60612

Phone: 312-996-1582

E-mail: edwardsb@uic.edu

Abstract Word Count: 162

Manuscript Word Count: 1038

Number of Tables: 0

Number of Figures: 3

Keywords: Impact, Landing, Reaction Force, Joint Moment, Inverse Dynamics 


\begin{abstract}
$\underline{\text { Abstract }}$
When performing inverse dynamics analysis, smoothing kinematic and force platform data at different cutoff frequencies creates an "impact" like artifact that is visible in the joint moments during impulsive activity. Here we illustrate a processing technique in which inverse dynamics analysis is performed on the raw kinematic and force platform data and the joint reaction forces and moments are subsequently smoothed based on the frequency content of the distal reaction force. The effectiveness of this technique is illustrated on forward dynamics simulation data with known intersegmental loads. We then apply the technique to an experimental data set of 10 subjects running at three prescribed speeds. We show that performing inverse dynamics on the raw data and subsequently smoothing the intersegmental loads results in minimal attenuation of the joint reaction force and avoids impact artifacts in the joint moments. Artifacts that occur using a traditional filtering technique are systematic, become more pronounced with speed, and are most noticeable at the hip joint.
\end{abstract}




\section{$\underline{\text { Introduction }}$}

Intersegmental forces and moments can provide insight into the internal loading of joints and coordination of movements during impulsive activity such as landing and running. Owing to high-frequency noise, movement artifact, and the need to double differentiate position data for segmental acceleration, it is necessary to smooth biomechanical data to accurately characterize intersegmental loads from inverse dynamics analysis. Traditionally, kinematic data are filtered at a low cutoff frequency (e.g., $8 \mathrm{~Hz}$ ) and force platform data are left raw or smoothed at a relatively high frequency (e.g., $50 \mathrm{~Hz}$ ) because of discrepancies in signal-to-noise-ratio [1-4]. It has been demonstrated that this approach creates an "impact" like artifact that is visible in joint moments during impact activity [5-7]. This artifact may result from the attenuation of equilibrating segmental accelerations in the inverse dynamics calculations $[6,7]$.

Joint moments free from artifacts can be resolved if kinematic and force platform data are smoothed at a relatively low, identical cutoff frequency (e.g., $20 \mathrm{~Hz}$ ) [5]. This approach is not ideal when reaction forces are of interest because the impact portion of the reaction force, which has major frequencies between 10 and $30 \mathrm{~Hz}$ during running [8], is attenuated. The purpose of this study is to illustrate a filtering technique in which inverse dynamics is performed on the raw kinematic and force platform data, and the resulting moments and reaction forces are subsequently smoothed based on the frequency content of the reaction force (similar to White and Podraza [7]). This technique is first validated on forward dynamics simulation data with known intersegmental loads [6]. Joint moments and reaction forces are then calculated for a group of ten subjects running at three selected speeds.

\section{$\underline{\text { Methods }}$}

\section{Simulation}


Running simulation data were obtained from the International Society of Biomechanics' data resource website (URL: http://isbweb.org/data/invdyn/index.html). These data were produced using a 2-D forward dynamics simulation, therefore the intersegmental loads are exactly known, and the associated kinematics are noise free [6]. To replicate a typical data collection system, the simulated kinematic and ground reaction force data were sampled at 160 and $1600 \mathrm{~Hz}$, respectively. White noise $(0.1 \mathrm{~mm}$ root mean square) was added to the kinematic data, which were then reinterpolated to $1600 \mathrm{~Hz}$ using a cubic-spline routine. A bottom-up, rigidbody inverse dynamics analysis was performed using two different filtering methods. In the first ( pre $_{\text {filt }}$ ), ground reaction forces were smoothed at $100 \mathrm{~Hz}$ and kinematics were smoothed at a cutoff frequency corresponding to the mean $98^{\text {th }}$ percentile frequency of all kinematic signals; inverse dynamics was then performed. In the second ( post $_{\text {filt }}$ ), inverse dynamics was performed on the raw data and the resulting joint reaction forces and moments were smoothed at the $99^{\text {th }}$ and $95^{\text {th }}$ percentile frequency of the distal vertical reaction force, respectively. Therefore, forces and moments at the ankle were filtered according to the frequency content of the vertical ground reaction force, the knee according to the vertical ankle reaction force, etc. For both methods, cutoff percentiles were chosen from optimization, and corresponded to the cutoff frequencies that minimized the average root mean squared error (RMSE) for each joint between inverse dynamics results and known intersegmental loads. Cutoff percentiles were calculated from the cumulative sum of an integrated power spectral density curve and the optimization process was performed 10 times with newly generated noise. A $4^{\text {th }}$ order, zero-lag Butterworth filter was used for both procedures.

Experimentation 
The experimentation described below has been previously reported [9]. Briefly, ten males (age $24.9 \pm 4.7 \mathrm{yrs}$; height $1.7 \pm 0.1 \mathrm{~m}$; mass $70.1 \pm 8.9 \mathrm{~kg}$ ) were recruited for this institutionally approved study after giving written informed consent. Subjects ran overground at 2.5, 3.5, and $4.5 \mathrm{~m} / \mathrm{s}$. Motion capture (Vicon MX, Vicon, Centennial, CO) and force platform (AMTI, Watertown, MA) data were collected concurrently at 160 and $1600 \mathrm{~Hz}$, respectively. Ten trials were performed at each speed. Inverse dynamics was used to calculate net-internal joint moments and reaction forces of the lower-extremity. Both pre filt $_{\text {and }}$ post $t_{\text {filt }}$ procedures were applied using the methods described above.

\section{$\underline{\text { Results }}$}

\section{Simulation}

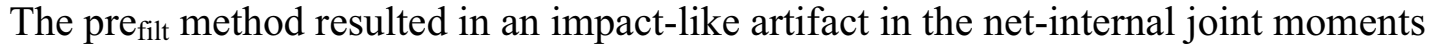
(Figure 1). RMSE progressively increased moving proximal towards the hip. The post filt $_{\text {method }}$ resulted in inverse dynamics that closely followed the known intersegemental loads; reaction forces were minimally attenuated and RMSEs were lower for the moments (Figure 1).

\section{Experimentation}

Similar results were observed for experimentally determined intersegmental loads. The pre $_{\text {filt }}$ method resulted in an impact like artifact in the hip and knee moment, and reaction forces were not attenuated using the post filt $_{\text {(Figure } 2 \text { ). Artifacts using the pre }}$ filt method became more pronounced with speed and these were visible in the group ensemble hip moment curves (Figure 3).

\section{$\underline{\text { Discussion }}$}

The impact artifacts presented here were largest at the hip. These could influence statistical outcomes aimed at quantifying differences in peak instantaneous magnitudes. 
Although these artifacts could be smoothed following inverse dynamics calculation, they are not randomly occurring events. They systematically increase with speed and are therefore highly related to the movement. Filtering these artifacts could potentially introduce systematic errors to the net internal joint moments.

When both joint reaction forces and moments are of interest, such is the case when analytical methods are used to estimate joint contact forces [10], van den Bogert and de Konging [6] recommended smoothing kinematic and kinetic data at identical cutoff frequencies, but performing inverse dynamics twice: once with a cutoff for optimal reaction forces, and again with a cutoff for optimal moments. This would likely produce similar results to the post filt $_{\text {t }}$

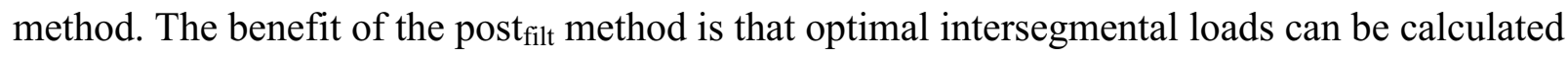
in a single inverse dynamics analysis, even if separate cutoff frequencies were chosen for each of three reaction forces and moments. For example, a lower cutoff percentile could be used for the hip reaction force to reduce the observed noise in Figure 2.

In summary, impact artifacts in joint moments are introduced when using traditional filtering techniques. These artifacts are most pronounced at faster speeds of running. Traditional filtering techniques would not be expected to introduce errors during less dynamic activities such as walking and stair climbing.

\section{Acknowledgements}

The authors thank Ton van den Bogert for making his inverse dynamics benchmark data available and for helpful discussions during manuscript preparation.

\section{Conflict of interest}

The authors have no conflict of interest 


\section{$\underline{\text { References }}$}

1. Arampatzis A, Brüggemann G-P, \& Metzler V. The effects of speed on leg stiffness and joint kinetics in human running. J Biomech (1999); 32, 1349-1353.

2. Edwards WB, Thomas JT, Gillette JC, Derrick TR. Internal femoral forces and moments during running: implications for stress fracture development. Clin Biomech [Bristol, Avon] (2008); 23, 1269-1278.

3. Ferber R, Davis IM, Williams III DS. Gender differences in lower extremity mechanics during running. Clin Biomech [Bristol, Avon] (2003); 18, 350-357.

4. Winter DA. Moments of forces and mechanical power in jogging. J Biomech (1983); 16, 9197.

5. Bisseling RW, Hof AL. Handling of impact forces in inverse dynamics. J Biomech (2006); 39, 2438-2444.

6. van den Bogert AJ, de Koning JJ. On optimal filtering for inverse dynamics analysis. In Proceedings of the IXth Biennial Conference of the Canadian Society for Biomechanics, Vancouver, BC, Canada. (1996); Retrieved Aug 4, 2010, from http://isbweb.org/c/isb/pub/files/orig_website/data/invdyn/csb96.pdf

7. White SC, Podraza JT. Processing effects on joint moments during impact landings. In Proceedings of the American Society of Biomechanics 31st Annual Meeting, Stanford, CA, USA. (2007); Retrieved Aug 4, 2010, from http://www.asbweb.org/conferences/2007/358.pdf

8. Boyer KA, Nigg BM. Muscle activity in the leg is tuned in response to impact force characteristics. J Biomech (2004); 37, 1583-1588. 
9. Edwards WB, Taylor D, Rudolphi TJ, Gillette JC, Derrick TR. Effects of running speed on a probabilistic stress fracture model. Clin Biomech [Bristol, Avon] (2010); 25, 372-377.

10. Glitsch U, Baumann W. The three-dimensional determination of internal loads in the lower extremity. J Biomech (1997); 30, 1123-1131. 


\section{Figure Captions}

Figure 1. Simulated inverse dynamics results (resultant magnitude of the reaction force and medial-lateral moment) and RMSE for the pre filt $_{\text {and }}$ post filt $_{\text {methods. Known instersegmental }}$ loads are shown in grey and the pre filt $_{\text {and }}$ post ${ }_{\text {filt }}$ methods are shown in black. Positive moments correspond to extension.

Figure 2. Experimentally determined intersegmental loads for a subject running at 2.5, 3.5, and $4.5 \mathrm{~m} / \mathrm{s}$. The pre filt $_{\text {method is shown in grey and the post }}$ filt method is shown in black. Positive forces are directed anterior, proximal, and lateral (dashed line $=$ anterior-posterior; solid line $=$ axial; dotted line $=$ medial-lateral). Positive moments correspond to adduction, internal rotation, and extension $($ dashed line $=$ anterior-posterior; dotted line $=$ torsion; solid line $=$ medial-lateral $)$.

Figure 3. Group ensemble experimentally determined joint moments for three speeds of running

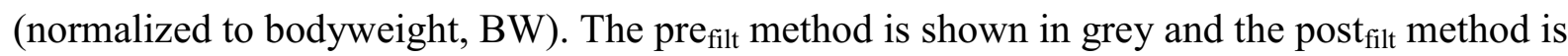
shown in black. Positive moments correspond to adduction, internal rotation, and extension (dashed line $=$ anterior-posterior; dotted line $=$ torsion; solid line $=$ medial-lateral $).$ 


\section{Figures}
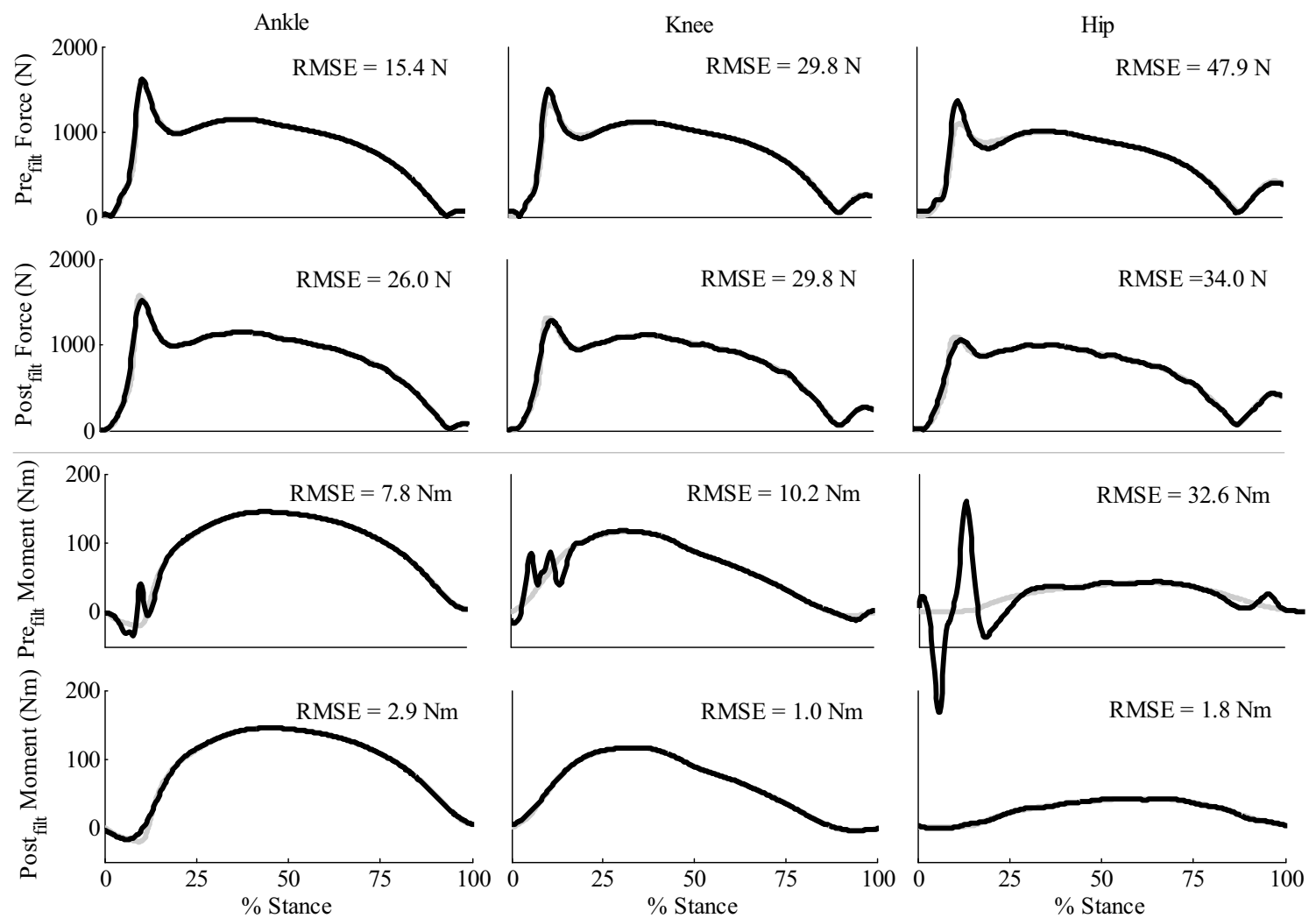

Figure 1. 
Figure 2.

$2.5 \mathrm{~m} / \mathrm{s}$
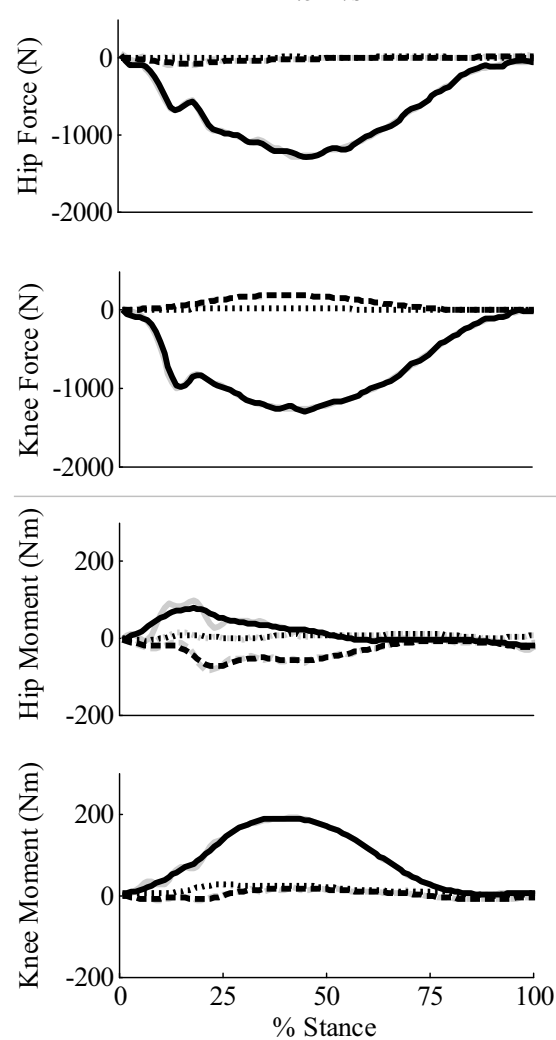

Figure 2.
$3.5 \mathrm{~m} / \mathrm{s}$
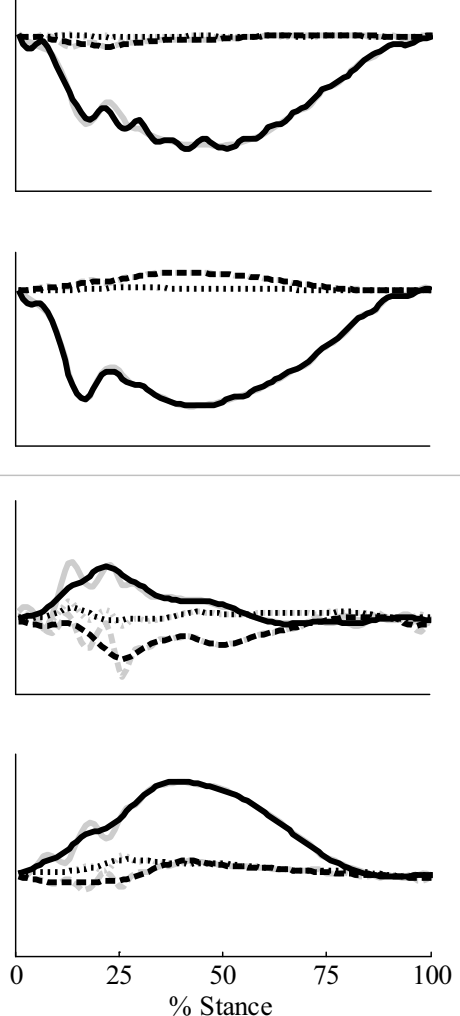

$4.5 \mathrm{~m} / \mathrm{s}$
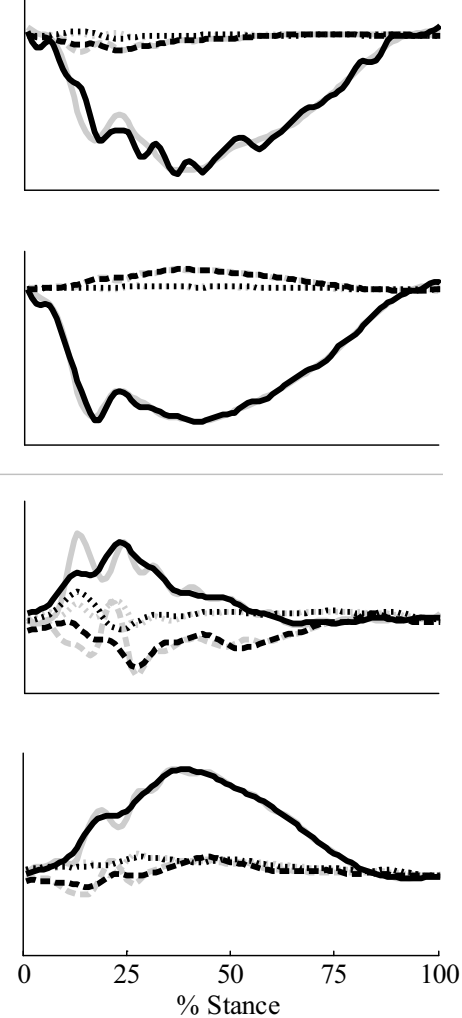

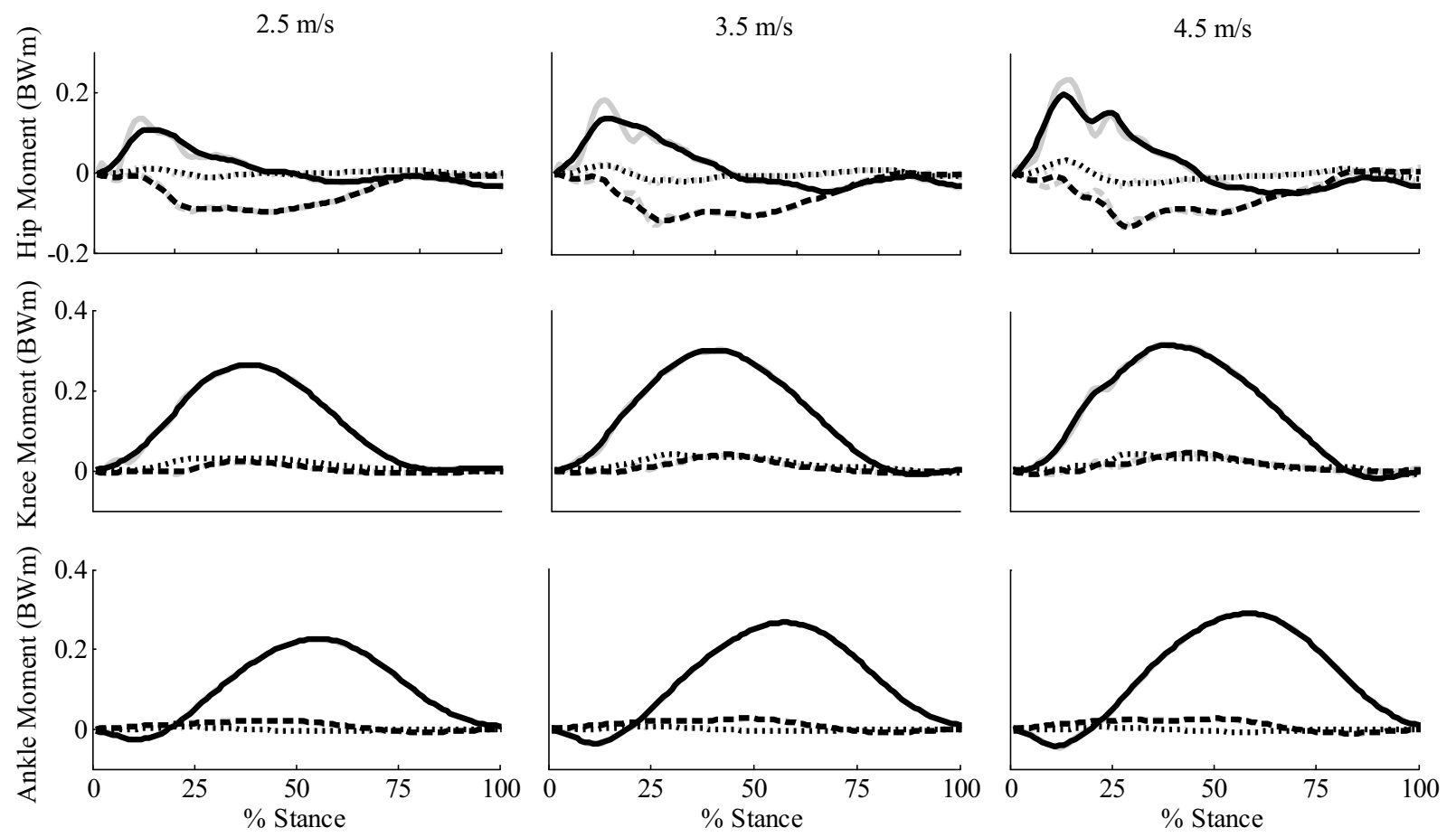

Figure 3. 